\title{
Is task interference in event-based prospective memory dependent on cue presentation?
}

\author{
SHAYNE LOFT \\ University of Queensland, Brisbane, Queensland, Australia \\ RebecCa Kearney \\ Griffith University, Brisbane, Queensland, Australia \\ AND \\ ROGER REMINGTON \\ University of Queensland, Brisbane, Queensland, Australia
}

\begin{abstract}
Whether having an intention produces a performance cost to ongoing activities (task interference) is central to theoretical claims regarding the mechanisms underlying cue detection in event-based prospective memory. Recent evidence suggests that task interference primarily reflects an attention allocation policy stored in memory when intentions are encoded. The present study examined whether these policies can change with ongoing task experience. In Experiment 1, task interference was more greatly reduced when expected cues were not presented than when they were. Experiment 2 replicated this effect when the importance of the prospective memory task was emphasized. In Experiment 3, task interference decreased with time, and this decrease was greater when expected cues were not presented than when they were. Cue presentation is crucial to maintenance of attention allocation policies established by task instructions. This is the first article to demonstrate changes in task interference with ongoing task experience without forewarning individuals of the relevance of upcoming ongoing task trials to intentions.
\end{abstract}

In recent years, researchers have shown increasing interest in memory tasks that require individuals to remember to perform an action at a designated point in the future. The term prospective memory (PM) is used to refer both to the tasks used and to the type of memory assumed to underlie performance. Most work conducted in this area has examined event-based PM, in which the occurrence of a PM cue signals that it is appropriate to perform an intended action. For example, in order to execute the delayed intention of stopping to buy medicine during the drive home from work, PM is required to recognize the cue (pharmacy) as relevant to the intention (to buy medicine), while attention is focused elsewhere (driving). Event-based PM laboratory paradigms simulate these conditions. Participants are required to perform a specific action - for instance, pressing the F1 key-upon cue presentation - for instance, the word donkey - while performing an ongoing task, such as rating words (Einstein \& McDaniel, 1990).

A current issue in the PM literature concerns the extent to which the processes underlying cue detection require centrally mediated attentional resources. According to the preparatory attention and memory (PAM) theory, cue detection always requires significant processing resources
(Smith \& Bayen, 2004; Smith, Hunt, McVay, \& McConnell, 2007). In support, PM task demands embedded in ongoing tasks slow down performance on noncue trials of ongoing tasks (e.g., Loft \& Yeo, 2007; Marsh, Hicks, Cook, Hansen, \& Pallos, 2003; Smith, 2003), an effect often referred to as task interference (Hicks, Marsh, \& Cook, 2005). Task interference is obtained on trials where PM cues do not occur, and thus does not reflect a cost associated with carrying out the intended action. In contrast, the multiprocess view argues that, under some task conditions, cue detection requires no resources and can occur automatically (Einstein \& McDaniel, 2005; McDaniel \& Einstein, 2007). In support, Einstein et al. (2005) found high rates of cue detection under some conditions where no evidence of task interference was found. The multiprocess view claims that specific task conditions determine the extent to which individuals are predisposed to rely on automatic retrieval processes to detect the PM cue, or instead allocate attentional resources to the process of cue detection.

The important point is that the presence (or absence) of the task interference effect is central to theoretical claims made by both PAM theory and the multiprocess view. Hence, there are theoretical ramifications for understand- 
ing the conditions under which the task interference effect will and will not be observed, and for specifying the cognitive mechanisms that give rise to it. One currently held view is that task interference primarily reflects an attention allocation policy stored in memory when intentions are encoded (Hicks et al., 2005; Marsh, Hicks, \& Cook, 2005). Since attention is presumed to be a limited-capacity resource, there can be an ongoing task-processing cost associated with allocating attention to the PM task, assuming that shared resources are involved. However, because ongoing task conditions can change from those expected when intentions are encoded, it would be advantageous if attention allocation policies could be adjusted with ongoing task experience, so that the degree to which intentions interfere with ongoing activities could be modified. For example, there are undoubtedly many ongoing real-world tasks for which people hold intentions for long periods during which expected PM cues are not encountered; it would be reasonable to expect that people might adjust their allocation of attention to ensure that ongoing tasks are not unduly compromised. Three studies are reported that test this premise by examining whether the magnitude of task interference is dependent on the presentation of PM cues in ongoing tasks. We approached this research question in the following way: Will intentions interfere less with ongoing tasks when expected cues are not presented than they will when expected cues are presented?

There is an emerging body of evidence to suggest that at least part of the task interference effect reflects an attention allocation policy that participants establish at the outset of the entire task set (Hicks et al., 2005; Marsh et al., 2005). According to proponents of this view, individuals assign a relative weighting of attention to the ongoing task and to the PM task. Support has come from showing that more difficult PM sets produce greater task interference. For example, poorly specified intentions (responding to a category of PM cues) cause greater task interference than do well specified intentions (responding to a specific cue; Hicks et al., 2005); and responding to multiple cues causes greater task interference than does responding to a single cue (Einstein et al., 2005) - presumably because participants believe that completing the former intentions will be more difficult. The attention allocation policy view is in line with the claims of the PAM theory that task interference reflects a single preparatory allocation of attention away from the ongoing task and toward the PM task (Smith $\&$ Bayen, 2004). According to PAM, preparatory attention serves the function of mapping ongoing task stimuli onto intentions, thus ensuring that a recognition check (the memory component of PAM) is initiated when a cue is presented. Other theoretical proposals in the literature are also consistent with the view that task interference is driven by a single parameter established at encoding. For example, Guynn (2003) proposed that individuals maintain their memory system in a retrieval mode, which she describes as a general preparedness or readiness to treat ongoing task stimuli as PM retrieval cues (cf. Tulving, 1983). These retrieval modes may involve increased levels of activation of cues that persist until intentions are complete, or until the goals and/or context of the task change (Goschke \& Kuhl,
1993; Lebiere \& Lee, 2001), thereby leaving less capacity for processing ongoing activity.

Given the number of and variety of our daily intentions, it may be unrealistic to expect all our unfilled intentions to be maintained at the state of readiness set by attention allocation policies at encoding. It is more likely that we deal with our multiple intentions by varying their activation according to their relevance to ongoing task contexts. This notion was supported by recent evidence suggesting that not only can task interference be reduced by warning participants on a trial-by-trial basis of the relevance of ongoing task stimuli to PM cue detection (Marsh, Cook, \& Hicks, 2006), but that it can even be eliminated by informing participants that cues will not be presented in an upcoming block of trials (Marsh, Hicks, \& Cook, 2006). For example, Marsh, Cook, and Hicks (2006) had participants link their intention to detect cues to a particular stimulus class (pictures vs. words) and found reductions in task interference when participants were reliably informed that the stimulus class about to be presented on the next trial (or block of trials) was irrelevant to cue detection. These findings are important, because they indicate that attention allocation policies are flexible and can be adjusted. However, it is critical to note that reductions in task interference demonstrated by Marsh, Cook, and Hicks (2006), and Marsh, Hicks, and Cook (2006) varied as a function of the extent to which participants were instructed in advance of the relevance of upcoming trials to cue detection. When Marsh, Cook, and Hicks (2006) presented picture and word stimuli randomly, no reduction in task interference was observed.

The present article extends the work of Marsh, Cook, and Hicks (2006) and Marsh, Hicks, and Cook (2006) by examining whether reductions in task interference can occur without forewarning individuals of the relevance of upcoming ongoing task trials to intentions, and in doing so, identifies a crucial factor that drives the maintenance of task interference. Specifically, we examine whether the allocation of attention to PM tasks, as presumably determined by attention allocation policies, will be reduced if these policies are not periodically reinforced by the presentation of PM cues. If this is the case, less task interference should occur when expected cues are not presented than when they are. Such an outcome would help clarify the extent to which everyday intentions might interfere with ongoing activity in everyday settings. As an example, consider an applied setting where PM is crucial, such as air traffic control (Dismukes, 2007; Loft, Humphreys, \& Neal, 2003): A controller may intend to assign an atypical flight altitude when accepting a Boeing 747 into his airspace because of an impeding thunderstorm. In addition to some ongoing tasks being more relevant to this intention than others (i.e., Marsh, Cook, \& Hicks, 2006; Marsh, Hicks, \& Cook, 2006), the frequency with which $747 \mathrm{~s}$ enter the sector could vary widely. There are also countless examples in everyday life of expected cues not being encountered: intending to give a message to several people at a party who never show up, for example. As argued further in the General Discussion, the outcomes of the present study also help inform future versions of the PAM model and the multiprocess view, since, at time of 
writing, these theories do not make definitive predictions about local adaptations in attention allocation to PM tasks with ongoing task experience.

As a precursor to the present study, Loft and Yeo (2007) found that task interference varied as a function of the frequency of the presentation of PM cues. However, lower probability cues were associated with greater average spacing between cues. Thus, participants in the Loft and Yeo study could have adopted a deliberate strategy, adjusting their attention allocation policies according to their observations of the average spacing between cue presentations. In this manner, cue spacing was an implicit indicator of the probability of cue presentation in upcoming ongoing task trials (although this indicator was not explicit, unlike those provided by Marsh, Cook, \& Hicks, 2006, and Marsh, Hicks, \& Cook, 2006). In contrast, participants in the PM no-cue group of the present study are never presented cues, and thus will have no data available regarding the "frequency" of cue presentation. Instead, they might think that they are missing cues and that they will subsequently need to keep their attention on the PM task. Given that cues will be learned to criterion at study, other participants may be confident that they are not missing any. However, given that these participants will be expecting up to eight cues to be presented during the ongoing task, it should be conceivable to them that cues will be bound to be presented "at any moment now," and thus that attention to the PM task should be maintained.

The present article describes three experiments. Experiment 1 provided an initial test of whether task interference would be reduced when expected PM cues were not presented, rather than when they were. Experiment 2 examined whether this reduction in task interference varied according to task importance manipulations. Of central interest here was whether task interference would still be significantly reduced when no cues were presented but when the importance of the PM task had been emphasized at encoding. Experiment 3 closely examined how task interference changed over the course of the ongoing task as a result of the presence or absence of cues and whether participants continue to allocate attention to the PM task after a long period without cue presentation.

\section{EXPERIMENT 1}

In Experiment 1, participants performed an ongoing lexical decision task. In addition, participants studied eight cue words and were instructed that they would be required to press the F1 key whenever presented with these words during the lexical decision task. These general task conditions have been proven to produce robust task interference effects (e.g., Loft \& Yeo, 2007; Marsh et al., 2005; Marsh et al., 2003; Smith, 2003). Participants completed a block of lexical decision trials alone, and received the $\mathrm{PM}$ instructions before completing a second block. The first block of lexical decision trials provided a within-subjects measure of baseline performance. There were two PM conditions in Experiment 1. In the PM cue condition, participants were presented with eight PM cues over the course of the ongoing task. In contrast, in the PM no-cue condition, participants were presented with none of the eight expected cues during the ongoing task. The purpose was to examine whether less task interference would occur on the second block of trials (compared with the baseline) when expected cues were not presented, compared with than when they were.

A further research question concerned whether holding event-based intentions interfered with the ongoing task when cues were not presented in the ongoing task. A control condition was included to address this question. Participants in the control condition performed two blocks of lexical decision trials but were not given the PM instruction. Data from the control condition were used to contrast the effects of embedding the PM task in the ongoing activity with the effects of performing the lexical decision task twice without the PM task.

\section{Method}

Participants. Sixty students enrolled in undergraduate psychology courses at the University of Queensland were paid A $\$ 10$ each for their participation. There were 20 participants in each condition. Participants in all three experiments were native English speakers.

Materials. The presentation of stimuli and collection of responses were accomplished through E-Prime (Schneider, Eschman, $\&$ Zuccolotto, 2002). A pool of 320 medium frequency words (4-8 letters in length) were randomly chosen from the 1994 issues of the Sydney Morning Herald (TSMH) word database (Dennis, 1995). The 320 nonwords were created by removing the first syllable of each word and placing it at the end of the letter string (Hunt \& Toth, 1990). Words and nonwords were randomly assigned to one of two lists (List 1 or List 2), creating two sets of 320 letter strings, each set containing 160 words and 160 nonwords. Two list orders were created: Order A, in which List 1 was presented in the first block of lexical decision trials and List 2 in the second block; and Order B, in which this order was reversed. Half the participants in each condition received the lists in Order A, half in Order B.

Eight words were randomly selected from Block 2 to serve as PM cues. Eight additional control words matched to cues on frequency and length were selected from TSMH word database. The order of presentation of trials was random except for the presentation of cues (or control words), which occurred on trials 20,60, 100, $140,180,220,260$, and 300 in Block 2. Cues (or control words) were presented in a random order. Participants in the PM-cue condition studied eight cue words at encoding, and were presented with these words in Block 2 of the lexical decision trials. The PM no-cue condition studied these same eight cue words, but were presented with eight control words in Block 2 of the lexical decision trials, and therefore were not presented with the cue words which they had studied. The control condition also studied eight cue words, and was presented with control words in Block 2.

Procedure. After consent was obtained, participants were informed that letter strings would be displayed on a computer screen and that they were required to decide as quickly and accurately as possible whether or not each string was a word. Responses were made by pressing one of the two home keys (D, word; K, nonword). Each trial contained three displays. The first display instructed participants to press the space bar to initiate the next trial. The second display was a focus point $(+)$ displayed in the center of the screen in black on a white background. The display time for each focus point was randomly selected from a set of possible display times (437, $500,562,625,687,750,822$, or $886 \mathrm{msec}$ ) to ensure that participants could not anticipate the appearance of letter strings. The third display was a string of letters which remained on the screen until the participant made an appropriate response. After these instructions had been read and understood, participants completed the 320 lexical decision trials that comprised Block 1 . On the completion of 
Block 1, participants were told they had completed the first half of the session, and that there would be a 3-min break before commencing the second half of the session.

Next, participants in the PM conditions were given PM instructions. The experimenter told participants that she was interested in their ability to remember to perform actions in the future. Participants were instructed that whenever a PM cue was detected, they should respond first to the lexical decision task and make their PM response during the subsequent waiting message between trials (Hicks et al., 2005; Loft \& Yeo, 2007; Marsh et al., 2005). Participants in the PM conditions were then given the eight cue words to study. Participants in the control condition also studied eight cue words, and were told that the purpose was to test their memory at the end of the session. Next, participants were given a word-stem memory test. In this test, participants were given the first one or two letters of each cue and were required to complete the words. Participants who could not accurately complete all cue words were asked to study the list again. Next, in order to avoid ceiling effects, participants completed a 5-min computer card task. After this retention interval, Block 2 of the lexical decision task was initiated for the PM conditions without further reference to the PM task. The control group was instructed to complete a second block of lexical decision trials. After completion, all participants were readministered the word-stem memory test.

\section{Results and Discussion}

An alpha level of .05 was used for all statistical tests. There was no significant difference between the three conditions in the proportion of PM cues recalled $(M=.92$, $S D=.11)$ on the final word-stem memory test $[F(1,57)=$ $\left.1.72, M S_{\mathrm{e}}=.02\right]$. PM performance was scored as the proportion of cue trials that received an F1 key response. Participants in the PM cue condition detected $0.73(S D=.23)$ of the cues presented in Block 2.

There was no evidence of task interference when the accuracy of lexical decisions was examined, and lexical decision accuracy was near ceiling $(96.3 \%)$. Thus, consistent with previous research that has examined task interference using lexical decisions (e.g., Hicks et al., 2005; Marsh et al., 2003), the average response time (RT) for word trials was the primary dependent measure. Several trials were excluded: (1) the initial four trials of each block; (2) trials that contained PM cues and noncue trials where the F1 key was incorrectly pressed (false alarms), to avoid response costs associated with performance of the intention (see Loft \& Yeo, 2007; Marsh et al., 2003); (3) the four trials that followed these aforementioned trials to avoid response costs associated with postoutput cue detection monitoring processes (see Einstein et al., 2005); (4) trials where RTs were greater than $3 S D$ s from a participant's grand mean; and (5) trials containing incorrect lexical decisions.

The data are summarized in Table 1. The first column, labeled Block 1, gives the average RT on word trials for Block 1 (baseline). The second column, labeled Block 2, gives the average RT on word trials for Block 2. The cost associated with the embedded PM tasks is evaluated by calculating individual task interference scores. For the two PM conditions, task interference scores reflected the change in RTs from Block 1, where the ongoing task was performed alone, to Block 2 where participants held intentions. Greater (more positive) task interference scores reflect a greater allocation of attention to the PM task. Task
Table 1

Average Time (in Milliseconds) to Respond to the Ongoing Task (and Task Interference) As a Function of Cue Presentation in Experiment 1

\begin{tabular}{lcccc}
\hline Cue Presentation & $N$ & Block 1 & Block 2 & Task Interference \\
\hline PM cue & 20 & 624 & 718 & +94 \\
PM no-cue & 20 & 618 & 647 & +29 \\
Control & 20 & 613 & 572 & -41 \\
\hline
\end{tabular}

Note-Control condition also included.

interference scores for the control group reflect the change in RT due to repeated performance of the ongoing task.

An ANOVA was conducted on RTs on Block 1. This analysis of baseline performance was not significant $(F<1)$. Thus, all three conditions were matched with respect to RTs on Block 1. As predicted, there was significantly less task interference when expected PM cues where not presented than when they were $[t(38)=2.83$, $p<.01]$. In this analysis, we excluded the 4 trials that followed cue trials and trials where the F1 key was pressed. Thus, this effect is unlikely to reflect postoutput cue detection monitoring processes allocated by participants in the PM cue condition. In order to decrease the likelihood of postoutput monitoring, we conducted a more stringent analysis by including only those noncue trials that were presented at least 30 trials after presentation of preceding cue trials (Trials 10-19; 50-59; 90-99; 130-139; etc.). In this analysis, there was significantly less task interference when expected cues were not presented $(+24 \mathrm{msec})$ than when they were $(+91 \mathrm{msec})[t(38)=2.29, p<.05] . .^{1}$ The task interference scores from this truncated analysis were highly comparable to those from the full task interference analysis presented in Table 1. Taken together, these findings demonstrate that the maintenance of attention allocation policies were at least partially dependent on the presentation of PM cues in the ongoing task.

The control condition demonstrated the expected practice effect with faster RTs on Block 2 than on Block 1 $[t(19)=3.54, p<.01]$. Task interference for the PM no-cue condition was significantly larger than the control condition $[t(38)=3.65, p<.01]$. Therefore, holding an intention still significantly interfered with the ongoing task, even when the PM cues were never presented.

\section{EXPERIMENT 2}

Experiment 1 provided evidence that task interference can be reduced under conditions where expected PM cues are not presented compared with PM conditions where they are presented. It is well documented that the relative importance of the PM task also influences the size of the task interference effect (Kliegel, Martin, McDaniel, \& Einstein, 2004; Loft \& Yeo, 2007; Smith \& Bayen, 2004). The increase in task interference with PM task importance is consistent with the idea that task interference reflects an attention allocation policy established at encoding, and that individuals assign a higher weighting of attention to the PM task at encoding when it is considered important (Hicks et al., 2005; Marsh et al., 2005). We conducted a second experiment to both rep- 
licate Experiment 1 and examine whether the relationship between cue presentation and task interference varied as a result of task importance manipulations.

Reduction of task interference in the absence of PM cues may be less pronounced when instructions emphasize the importance of the PM task than when they emphasize the importance of the ongoing task. When the PM task is important, participants may be more likely to maintain their attention allocation policies because of the perceived importance of detecting cues, thereby keeping attention allocated to the PM task, despite the absence of cues. In contrast, when the ongoing task is important, participants may be less willing to compromise performance on it, thus less likely to continue allocating attention to the PM task in the absence of cues (as in Experiment 1). If so, there should be an interaction between importance and cue presentation, in that the difference in task interference between the PM no-cue and PM cue conditions will be less pronounced when instructions emphasize the importance of the PM task than when they emphasize the importance of the ongoing task.

Alternatively, modifications to attention allocation policies made with ongoing task experience may have a strong, perhaps automatic, adaptation component that responds to cue absence similarly, despite the stated importance of tasks. Thus, the reduction in attention allocation to PM tasks when expected cues are not encountered in ongoing tasks may be the same regardless of the initial relative importance of the tasks. If this is the case, an interaction between importance and PM cue presentation would not be expected. In addition, we should find a significant difference in task interference between the PM cue and PM no-cue conditions when the importance of the PM task has been emphasized, indicating that the maintenance of attention allocation policies is dependent to some degree on the presentation of cues, even when the importance of detecting cues has been previously stressed to participants.

\section{Method}

Participants. A total of 83 students enrolled in undergraduate psychology courses at the University of Queensland were paid A $\$ 10$ each for their participation (cell Ns are given in Table 2). Two participants were excluded because of extremely high false alarm rates to the PM task component ( $>136$ false alarms).

Materials and Procedure. Materials and procedures were identical to those in Experiment 1, with the addition of task importance instructions. After testing for cue recollection, the experimenter verbally delivered the task importance instructions, emphasizing the importance of either the PM task or the ongoing task (Loft \& Yeo, 2007; Smith \& Bayen, 2004).

\section{Results and Discussion}

A 2 (importance) $\times 2$ (cue presentation) ANOVA was conducted on the number of PM cues recalled on the final memory test. The proportion of cues recalled $(M=.92$, $S D=.13)$ was not affected by importance $(F<1)$ or cue presentation $(F<1)$, and there was no interaction $\left[F(1,77)=2.29, M S_{\mathrm{e}}=.04\right.$, n.s. $]$. A directional independent groups $t$ test revealed that participants detected more cues when the PM task was important $(M=.78, S D=$ .17) than when the ongoing task was important $(M=.64$, $S D=.27)[t(38)=1.92, p<.05]$. There was no evidence of task interference when the accuracy of lexical decisions was examined, and lexical decision accuracy was near ceiling $(95.4 \%)$.

The RT data are summarized in Table 2. Planned comparisons conducted on RTs in Block 1 revealed no significant effects (all $p \mathrm{~s}>.10$ ). Thus, the four PM conditions were matched with respect to RTs on Block 1. The cost associated with the PM task was again evaluated by calculating individual task interference scores. Task interference scores were analyzed with a 2 (importance) $\times 2$ (cue presentation) ANOVA. Consistent with previous research (Loft \& Yeo, 2007; Smith \& Bayen, 2004), there was significantly less task interference when the ongoing task was important $(+39 \mathrm{msec})$ than when the PM task was important $(+112 \mathrm{msec})\left[F(1,77)=20.20, M S_{\mathrm{e}}=105,147.95\right.$, $p<.01]$. Consistent with Experiment 1, there was significantly less task interference when expected cues were not presented $(+50 \mathrm{msec})$ than when they were $(+101 \mathrm{msec})$ $\left[F(1,77)=9.85, M S_{\mathrm{e}}=51,279.91, p<.01\right]$.

There was no evidence at all of an interaction between importance and cue presentation $(F=.02)$. Inspection of task interference data in Table 2 indicates that differences in task interference between the PM cue and PM no-cue conditions were highly similar, regardless of the relative importance of the two tasks. Furthermore, a follow-up simple-effects test revealed that there was significantly less task interference under PM no-cue conditions than under PM cue conditions, when the PM task was impor$\operatorname{tant}[t(39)=2.03, p<.05]$. Even when the importance of detecting PM cues had been stressed to participants, the maintenance of attention allocation policies was at least partially dependent on the presentation of cues. The data indicated that task importance manipulations did not affect the sensitivity of attention allocation policies to the absence of cues. This is consistent with the notion thatin addition to deliberate strategies of attention allocation set at encoding as a result of importance manipulationsmodifications in attention allocation to the PM task made

Table 2

Average Time (in Milliseconds) to Respond to the Ongoing Task (and Task Interference) As a Function of Cue Presentation and Task Importance in Experiment 2

\begin{tabular}{|c|c|c|c|c|c|c|c|c|}
\hline \multirow[b]{3}{*}{ Cue Presentation } & \multicolumn{8}{|c|}{ Importance } \\
\hline & \multicolumn{4}{|c|}{ PM Important } & \multicolumn{4}{|c|}{ Ongoing Important } \\
\hline & $N$ & Block 1 & Block 2 & $\begin{array}{c}\text { Task } \\
\text { Interference }\end{array}$ & $N$ & Block 1 & Block 2 & $\begin{array}{c}\text { Task } \\
\text { Interference }\end{array}$ \\
\hline PM cue & 20 & 569 & 707 & +138 & 20 & 607 & 670 & +63 \\
\hline PM no-cue & 21 & 595 & 680 & +85 & 20 & 579 & 594 & +15 \\
\hline
\end{tabular}


with ongoing task experience may have a strong adaptation component that responds to cue absence similarly, despite this stated importance.

As in Experiment 1, we conducted a follow-up analysis for noncue trials that were presented a minimum of 30 trials after the presentation of PM cue trials. A 2 (importance) $\times 2$ (cue presentation) ANOVA revealed a significant main effect for task importance (LD important +40 msec vs. PM important $+111 \mathrm{msec})[F(1,77)=$ $\left.18.07, M S_{\mathrm{e}}=99,317.56, p<.01\right]$. The main effect for cue presentation was also replicated (PM no-cue +49 msec vs. PM cue $+102 \mathrm{msec})\left[F(1,77)=10.55, M S_{\mathrm{e}}=57,975.26\right.$, $p<.01]$. There was again no evidence of an interaction $(F=.10)$. Finally, the follow-up simple effects test again indicated significantly less task interference for the PM no-cue condition than for the PM cue condition when the PM task was important $[t(39)=2.22, p<.05]$. In sum, the task interference scores from this truncated analysis were highly comparable to those from the full task interference analysis presented in Table 2 .

\section{EXPERIMENT 3}

The primary goal of Experiment 3 was to examine how attention allocation policies change over the course of the ongoing task as a result of the presence or absence of cues. In the previous experiments, we made the reasonable assumption that attention allocation policies set by participants in the PM cue and PM no-cue conditions at encoding would be highly similar, because they received identical task instructions. One explanation for the effects of cue presentation on task interference, then, is that subsequent attention allocation to the PM task decreased when no expected cues were presented, and, in contrast, was maintained at the same level set at encoding when expected cues were presented. However, Einstein et al. (2005) found evidence that attention allocation to PM tasks can also decrease over the course of the ongoing task when expected cues are presented.

In the previous experiments the first PM cue was presented to the PM cue condition after only 20 trials. Given that the first four trials and all nonwords were excluded, the remaining valid trials were unlikely to provide a reliable measure of baseline attention allocation policies. In Experiment 3, the PM cue and PM no-cue conditions were both presented a larger block of 100 trials (Block 1) without cue presentation, in order to obtain a reliable estimate of baseline attention allocation policies. RTs for these two PM conditions in Block 1 should be highly similar, and both should be larger than RTs for the control condition. After the presentation of Block 1, participants were presented a second block of 540 trials. In Block 2, participants in the PM cue condition were presented cues, whereas those in the PM no-cue condition were not. In a final block of 100 trials (Block 3), no cues were presented for either PM condition. Block 1 provides a more robust baseline measure of attention allocation policies than did previous experiments. Block 3 provides a subsequent measure of these policies after a period of time with or without cue presentation.
Differences in RTs between Block 3 and Block 1 (task interference change scores) reflects changes in attention allocation to the PM task as a result of the presence or absence of cues in Block 2. Participants in a control condition performed the entire lexical decision task without PM instruction, providing a suitable single-task baseline.

Einstein et al. (2005) found reduced task interference for participants who held intentions as a function of time spent on the ongoing task, indicating that attention allocation to PM tasks can decrease over the course of the ongoing task, regardless of cue presentation. On this basis, we predicted that attention allocation to the PM task by participants in the PM conditions would decrease over the course of the ongoing task. If this is the case, task interference change scores should be greater for the PM conditions than for the control condition. Also, on the basis of findings from Experiments 1 and 2, we predicted that this decrease in attention allocation to the PM task over the course of the ongoing task would be larger when participants were not presented cues than when they were. If this is the case, task interference change scores should be larger for the PM nocue condition than for the PM cue condition.

Although task interference for the PM no-cue conditions was significantly reduced in Experiment 1, it was not entirely eliminated. In Experiment 3, we presented a fairly large second block of trials in order for the effects of time on task interference - with or without the presence of PM cues - to be more fully realized. A secondary goal of Experiment 3 was to examine whether participants in the PM nocue conditions continued to allocate attention to the PM task after this significantly long period without cue presentation (Block $1+$ Block $2=640$ trials without cue presentation). If attention is still allocated, RTs in Block 3 should be larger for the PM no-cue condition than the control condition.

\section{Method}

Participants. A total of 93 students enrolled in undergraduate psychology courses at the University of Queensland participated in return for course credit (cell Ns are given in Table 3).

Materials. The same pool of 320 words and 320 nonwords used in previous experiments was presented. An additional 50 words (4-8 letters in length) were randomly chosen from TSMH word database (Dennis, 1995), and 50 nonwords were created using the Hunt and Toth (1990) method. A random subset of these words and nonwords were assigned to List 2, creating one set of 540 letter strings with each set containing 270 words and 270 nonwords. List 2 was always presented in the second block of lexical decision trials. The remaining words and nonwords were randomly assigned to one of two lists (List 1 and List 3), creating two sets of 100 letter strings, each set containing 50 words and 50 nonwords. Two list orders were created: Order A, in which List 1 was presented in the first block of lexical decision trials and List 3 in the third block; and Order B, in which this order was reversed. Half the participants in each condition received the lists in Order A, half in Order B.

Eight words were randomly selected from List 2 to serve as PM cues in Block 2. The same 8 additional control words used in Experiments 1 and 2 were used. The order of presentation of trials was random except for the presentation of cues (or control words), which occurred on Trials 10, 50, 90, 130, 170, 210, 250, 290, 330, 370, 410, 450,490 , and 530 in Block 2. Six cues (or 6 control words) were presented twice each in Block 2, and the remaining 2 cues (or 2 control words) once each. Cues (or control words) were presented in random order, with each cue (or control word) presented once before any cue 
Table 3

Average Time (in Milliseconds) to Respond to the Ongoing Task

(and Task Interference Change Scores) As a Function of Cue Presentation in Experiment 3

\begin{tabular}{lccccc}
\hline $\begin{array}{c}\text { Cue } \\
\text { Presentation }\end{array}$ & $N$ & $\begin{array}{c}\text { Block 1 } \\
(100 \text { trials })\end{array}$ & $\begin{array}{c}\text { Block 2 } \\
(540 \text { trials })\end{array}$ & $\begin{array}{c}\text { Block 3 } \\
(100 \text { trials })\end{array}$ & $\begin{array}{c}\text { Task Interference } \\
\text { Change Scores }\end{array}$ \\
\hline PM cue & 32 & 777 & 770 & 735 & -42 \\
PM no-cue & 31 & 761 & 727 & 663 & -98 \\
Control & 30 & 616 & 617 & 611 & -5 \\
\hline
\end{tabular}

Note-Control condition also included; Task Interference Change Scores, Block 3 minus Block 1.

or control word was repeated. Participants in the PM cue condition studied 8 cue words and were presented with a total of 14 cues in Block 2 of the lexical decision trials. The PM no-cue condition studied these same 8 cue words, but were presented with control words in Block 2, and were therefore not presented with the cue words which they had studied. The control condition also studied 8 cue words and was presented with control words in Block 2.

Procedure. After the lexical decision instructions, participants in the PM conditions were given PM instructions identical to instructions given in previous experiments, except that participants were told that the eight cues "may be presented more than once each" during the ongoing task. Participants in the PM conditions were then given the eight cue words to study. Participants in the control condition also studied the eight cue words, and were told that the purpose was to test their memory at the end of the session. Participants were given two minutes to study these words (a word-stem memory test could not be conducted due to time restrictions). After a 5-min computer card task, the lexical decision trials were initiated, without further reference to the PM task. All three blocks of lexical decisions were performed without break. After completion, all participants completed the wordstem memory test for the cues.

\section{Results and Discussion}

There was no significant difference between the three conditions in the proportion of PM cues recalled $(M=.79$, $S D=.23)$ on the final memory test $(F<1)$. Participants in the PM cue condition detected $0.70(S D=.25)$ of cues presented in Block 2. As in previous experiments, lexical decision accuracy was near ceiling $(95.6 \%)$. However, a 3 (group: PM cue, PM no-cue, control) $\times 2$ (block: 1 or 3 ) mixed ANOVA revealed that participants were more accurate in Block $1(M=.96, S D=.03)$ than in Block $3(M=$ $.95, S D=.04)\left[F(2,90)=17.04, M S_{\mathrm{e}}=.01, p<.01\right]$. This reduction in lexical decision accuracy with time is possibly due to the fatigue induced by the unusual length of the lexical decision task. There was no evidence of an interaction between group and block $(F<1)$. Although the PM conditions were less accurate in Block 3 than in Block 1, the control condition was equally inaccurate, so there is no evidence that this decrease in accuracy for participants in PM conditions reflected changes in task interference. Most importantly, the decrease in accuracy for participants in the PM cue and PM no-cue conditions did not significantly differ.

The RT data are summarized in Table 3. The first column, labeled Block 1, gives the average RT on word trials for Block 1, where no cues were presented, but for which the intention to respond to PM cues was active for the PM conditions. RTs at Block 1 provide a reliable baseline measure of attention allocation policies before cue presentation. As expected, there were no differences between the
RTs of the PM cue and PM no-cue conditions in Block 1 $(t<1)$, indicating that participants in the two conditions set a similar weight of attention to the PM task at encoding (this comes at no surprise, since participants in the PM cue and PM no-cue conditions received identical task instructions). As predicted, RTs in Block 1 were significantly higher for both the PM cue condition $[t(60)=4.48$, $p<.01]$ and the PM no-cue condition $[t(59)=5.34, p<$ .01] than for the control condition. Participants in both PM conditions were allocating a significant amount of attention to the PM task in Block 1.

The second column in Table 3, labeled "Block 2," gives the average RT on word trials for Block 2, where cues were presented to the PM cue condition but not the PM no-cue condition. The third column, labeled "Block 3," gives the average RT for word trials for Block 3 when neither PM condition was presented cues. Task interference change scores were calculated by subtracting RTs in Block 3 from RTs in Block 1. More negative changes scores reflect greater changes in allocation of attention to the PM task from Block 1 to Block 3 as a result of the presence or absence of cues in Block 2.

In contrast to Experiment 1, the control condition did not demonstrate a practice effect $(t<1)$, with RTs to Block 1 comparable to those to Block 3. When taken together with the decrease in accuracy from Block 1 to Block 3, this suggests that performing 740 lexical decisions without break was fatiguing. However, as predicted, task interference change scores were significantly greater for the PM conditions than for the control condition $[t(91)=2.96, p<.01]$, indicating that attention allocation to the PM tasks significantly decreased over the course of the ongoing task for the PM conditions (consistent with Einstein et al., 2005). In addition, these change scores were significantly greater for the PM no-cue condition than for the PM cue condition $[t(61)=2.04, p<.05]$, indicating that this decrease in attention allocation to the PM task was greater for participants not presented cues than for participants presented cues. Finally, RTs in Block 3 were significantly greater for the PM no-cue condition than for the control condition $[t(59)=2.25, p<.05]$. Participants were still allocating attention to the PM task in Block 3, even after 640 trials (Blocks 1 and 2) without a single cue presentation.

\section{GENERAL DISCUSSION}

The general message of these experiments is that task interference can be at least partially dependent on the pre- 
sentation of PM cues in ongoing tasks. In Experiment 1, less task interference was found when expected cues were not presented than when they were presented. This effect of cue presentation was replicated in Experiment 2, despite the importance of detecting cues being emphasized at encoding. In Experiment 3, task interference decreased over the course of the ongoing task, a decrease in task interference that was larger for participants who had not been presented cues than for participants who had. However, although task interference for participants not presented cues was significantly reduced, it was never entirely eliminated, even after a fairly long period without cue presentation. To our knowledge, the present article is the first to report reductions in task interference without forewarning participants of the relevance of upcoming trials to intentions. In the remainder of this discussion, we provide an evaluation of the theoretical implications of these findings.

Something of a consensus has recently developed around the idea that task interference reflects an attention allocation policy (Hicks et al., 2005; Marsh et al., 2005; Marsh, Cook, \& Hicks, 2006), preparatory attention (Smith \& Bayen, 2004), or instantiation of retrieval mode (Guynn, 2003) that is established at encoding and that divides a limited pool of resources between the ongoing task and the PM task. In the present study, participants in the PM cue and PM no-cue conditions would be expected to have set highly similar attention allocation policies (or preparatory attention, retrieval modes) because they received identical task instructions, and this was confirmed by the analysis of RTs in Block 1 of Experiment 3. We reasoned that the potential to adjust policies set at encoding would be advantageous, because conditions in ongoing task environments can invariably change from those initially expected (Hicks et al., 2005). In support of this proposition, the present data suggest that attention allocation policies can be adjusted with experience in ongoing task environments where no expected PM cues are presented. The finding that task interference decreased over the course of the ongoing task even when cues were periodically presented and mostly detected is consistent with the idea that it is difficult to maintain controlled self-regulatory processes over extended periods of task time (see Bargh \& Chartrand, 1999; McDaniel \& Einstein, 2007). Furthermore, the present data demonstrated that the allocation of attention to PM tasks, as set by attention allocation policies at encoding, can decrease at an even faster rate over the course of the ongoing task if these policies are not periodically reinforced by the presentation of cues.

At the outset of these studies, there were two reasons why it was not immediately clear whether task interference would be reduced when expected PM cues were not presented rather than when they were presented. First, participants were not explicitly (Marsh, Cook, \& Hicks, 2006; Marsh, Hicks, \& Cook, 2006) or implicitly (Loft $\&$ Yeo, 2007) forewarned of the relevance of imminent ongoing task trials to intentions; they should, therefore, have expected cues to be presented on any upcoming trials, or felt that they had been missing cues. Second, even if participants were confident that they had not missed cues, the expectation should have been strong that eight cues learned to criterion would eventually be presented, particularly in Experiment 2, when the experimenter had emphasized the importance of detecting cues. Despite these aforementioned factors, the task interference data suggest that participants not presented cues developed over the course of the task an expectation that the probability of cue presentation (or the temporal imminence of cue presentation) was different to what they had expected when forming their attention allocation policy at encoding. Thus, attention allocation policies set by participants in PM task paradigms can be adapted on the basis of the local history of the ongoing task environment. This proposal is consistent with many theoretical approaches to human decision making, including theories of information foraging (Pirolli \& Card, 1999), reinforcement learning (Estes, 1950), and more general theories of bounded rationality (Simon, 1956) and human cognition (Anderson, 1991), all of which share the general assumption that humans can adapt their behavior on the basis of prior contextual experience in specific tasks.

The findings - that holding an intention still interfered with the ongoing task when PM cues were not presented, and even after cues had not been presented over a large number of ongoing task trials - suggest that a significant portion of attention to PM tasks was fixed for the duration of the ongoing task period for which the intention to detect cues was relevant. These findings are consistent with PAM theory and proposals in the literature that PM representations (such as those instantiated by retrieval modes; Guynn, 2003) hold a special status in memory that persists at some level until intentions are complete or until there is a change in the goals and/or context of tasks (Goschke \& Kuhl, 1993; Lebiere \& Lee, 2001). Moreover, it indicates that these special-status memory items persist in representations that affect task processing; that is, they share limited-capacity resources with task operations.

The PAM model is a multinomial model for cue detection and is therefore not intended to predict actual RT values. Nevertheless, the preparatory attention parameter can be used to reliably estimate general differences in task interference observed as a function of task factors (such as cue presentation). For example, Smith and Bayen (2004) found that manipulating the categorical similarity between cues and noncues increased the preparatory attention parameter estimate and task interference. However, local adaptations in the allocation of preparatory attention over the course of ongoing tasks cannot currently be captured by the PAM model, since it has a single preparatory attention parameter that is set to be equal for all trial types (see Smith \& Bayen, 2004, p. 759). Nonetheless, PAM represents a good place to start tackling the issues raised by the present article and the Marsh, Cook, and Hicks (2006) article within a formal mathematical modeling framework. For example, PAM could model the Marsh, Cook, and Hicks (2006) data by using two preparatory attention parameters, one for relevant trials and one for irrelevant trials. In contrast, to capture the variations in preparatory attention that occurred from Block 1 to Block 3 in Experiment 3 , the preparatory attention parameter would need to 
allow preparatory attention estimates to vary according to some function of the history of experienced task conditions and the general passage of time.

According to the multiprocess view, there are several specific task parameters (e.g., strength of association between PM cues and responses; saliency of cues; focal processing) that determine whether a given context is likely to encourage automatic cue detection, and thus whether individuals are likely to allocate attention to the PM task (McDaniel \& Einstein, 2007). Given that participants generally have knowledge of such conditions at the time task instructions are given, the multiprocess framework's interpretation of task interference is similar to the attention allocation policy view, insofar as it assumes that participants, after being instructed of the task conditions to be expected, decide how much attention to the PM task will be required for cue detection. However, in a situation in which cues are unexpectedly but consistently presented in a salient manner (e.g., red font), the multiprocess view would presumably predict that participants would be able to rely more on automatic retrieval processes and thus reduce (or eliminate entirely) their attention to the PM task (but see findings of Smith et al., 2007). However, there are no mechanisms specified in the current version of the multiprocess view that would account for such local adaptations in attention allocation to PM tasks with ongoing task experience.

The present findings also have implications for understanding real-world situations that may be especially prone to PM failure. PAM theory argues that preparatory attention is functionally related to PM performance. To the extent that preparatory attention is required for cue detection, the probability of cue detection should decrease with time spent in relevant ongoing task contexts (see Einstein et al., 2005), and this decrease in cue detection should be particularly large when no expected cues have been encountered. However, task interference does not always guarantee that cues will be better detected (Loft \& Yeo, 2007; Marsh et al., 2005), because this depends on how attention resources are deployed and whether those resources are actually useful to cue detection. It is appropriate at this point to be clear that the present article was not designed to become part of the debate between PAM theory and the multiprocess view regarding whether PM retrieval can be totally resource free; nonetheless, the multiprocess view would argue that attention to a cue can lead to automatic cue detection under certain conditions, and that - although participants may allocate attention to the PM task because they think that it is required - the passage of time, or lack of cue presentation, may actually have no effect on cue detection.

On a final note, it must be acknowledged that it is difficult to pinpoint the precise nature and function of the cognitive mechanisms that give rise to task interference (for some discussion, see Hicks et al., 2005; Loft, Humphreys, $\&$ Whitney, in press). PAM theory claims that preparatory attention serves the function of mapping ongoing task stimuli onto intentions, thus promoting the recognition of cues (Smith et al., 2007). However, in addition to preparatory attention and other processes such as retrieval modes (Guynn, 2003), at least part of the task interference effect can potentially be driven by the stimulus. One mechanism that has been proposed is some form of poststimulus processing check (Guynn, 2003; Marsh \& Hicks, 1998). In addition, part of the task interference effect may be created by a sense of significance or familiarity with stimuli that initiates a memory retrieval process required to identify a cue (Loft et al., in press; McDaniel, Guynn, Einstein, \& Breneiser, 2004). The fact that Marsh, Cook, and Hicks (2006) did not observe reductions in task interference for irrelevant trials randomly mixed with relevant trials does not support the poststimulus processing view. In contrast, we cannot rule out poststimulus processing, because the word stimuli we used were all relevant to cue detection.

In conclusion, these three experiments demonstrated that task interference was dependent on the presentation of PM cues, indicating that cue presentation affected the extent to which attention was recruited away from the ongoing task and allocated to the PM task. In this article, we have argued for the existence of some mechanism that adjusts the allocation of attention to PM tasks on the basis of task experience. We propose that this adaptive mechanism serves to protect ongoing tasks as much as possible from disruption, without comprising the flexibility that allows the rapid execution of intended actions when appropriate. Continued investigation into the reciprocal relationship between cue presentation and task interference is likely to be an important theoretical endeavor, and may provide insight into how individuals prevent long-term intentions from interfering with everyday and professional work activities.

\section{AUTHOR NOTE}

We thank Freya Combes, Emerald Quinn, Lara Campbell, and Rebecca Rainbow for their dedicated help in collecting and preparing the data. We also thank Gillian Yeo for commenting on an earlier draft. Correspondence concerning this article should be addressed to S. Loft, School of Psychology, University of Queensland, Queensland 4072, Australia (e-mail: lofts@psy.uq.edu.au).

\section{REFERENCES}

ANDERSON, J. R. (1991). Is human cognition adaptive? Behavioral \& Brain Sciences, 14, 471-517.

BARGH, J. A., \& Chartrand, T. L. (1999). The unbearable automaticity of being. American Psychologist, 54, 462-479.

DenNis, S. (1995). The impact of the environment on the word frequency and null list strength effects in recognition memory. Noetica: Open Forum, 1. Retrieved March 5, 2004, from psy.uq.edu $. \mathrm{au} / \mathrm{Cog}$ Psych/Noetica.

Dismukes, R. K. (2007). Prospective memory in aviation and everyday settings. To appear in M. Kliegel, M. A. McDaniel, \& G. O. Einstein (Eds.), Prospective memory: Cognitive, neuroscience, developmental, and applied perspectives. Mahwah, NJ: Erlbaum.

Einstein, G. O., \& McDaniel, M. A. (1990). Normal aging and prospective memory. Journal of Experimental Psychology: Learning, Memory, \& Cognition, 16, 717-726.

Einstein, G. O., \& McDaniel, M. A. (2005). Prospective memory: Multiple retrieval processes. Current Directions in Psychological Science, 14, 286-290.

Einstein, G. O., McDaniel, M. A., Thomas, R., Mayfield, S., Shank, H., Morrisette, N., \& Breneiser, J. (2005). Multiple processes in prospective memory retrieval: Factors determining monitoring versus spontaneous retrieval. Journal of Experimental Psychology: General, 134, 327-342.

Estes, W. K. (1950). Toward a statistical theory of learning. Psychological Review, 57, 94-107.

Goschke, T., \& Kuhl, J. (1993). Representation of intentions: Per- 
sisting activation in memory. Journal of Experimental Psychology: Learning, Memory, \& Cognition, 19, 1211-1226.

GUYNN, M. J. (2003). A two-process model of strategic monitoring in event-based prospective memory: Activation/retrieval mode and checking. International Journal of Psychology, 38, 245-256.

Hicks, J. L., Marsh, R. L., \& CoOK, G. I. (2005). Task interference in time-based, event-based, and dual intention prospective memory conditions. Journal of Memory \& Language, 53, 430-444.

Hunt, R. R., \& ToTH, J. P. (1990). Perceptual identification, fragment completion, and free recall: Concepts and data. Journal of Experimental Psychology: Learning, Memory, \& Cognition, 16, 282-290.

Kliegel, M., Martin, M., McDaniel, M. A., \& Einstein, G. O. (2004). Importance effects on performance in event-based prospective memory tasks. Memory, 12, 553-561.

Lebiere, C., \& LeE, F. J. (2001). Intention superiority effect: A contextsensitivity account. In E. M. Altmann, A. Cleeremans, C. D. Schunn, \& W. D. Gray (Eds.), Proceedings of the 2001 Fourth International Conference on Cognitive Modeling (pp. 139-144). Mahwah, NJ: Erlbaum.

Loft, S., Humphreys, M., \& Neal, A. (2003). Prospective memory in air traffic control. In G. Edkins \& P. Pfister (Eds.), Innovation \& Consolidation in Aviation, pp. 287-293. Aldershot, U.K.: Ashgate.

Loft, S., Humphreys, M., \& Whitney, S. (in press). Control of access to memory: The use of task interference as a behavioural probe. Journal of Memory \& Language.

LofT, S., \& YEo, G. (2007). An investigation into the resource requirements of event-based prospective memory. Memory \& Cognition, 35, 263-274.

MARsh, R. L., CooK, G. I., \& Hicks, J. L. (2006). Task interference from event-based intentions can be material specific. Memory \& Cognition, 34, 1636-1643.

MARSH, R. L., \& Hicks, J. L. (1998). Event-based prospective memory and executive control of working memory. Journal of Experimental Psychology: Learning, Memory, \& Cognition, 24, 336-349.

MARSH, R. L., Hicks, J. L., \& CoOK, G. I. (2005). On the relationship between effort toward an ongoing task and cue detection in event-based prospective memory. Journal of Experimental Psychology: Learning, Memory, \& Cognition, 31, 68-75.

Marsh, R. L., Hicks, J. L., \& CoOK, G. I. (2006). Task interference from prospective memories covaries with contextual associations of fulfilling them. Memory \& Cognition, 34, 1037-1045.

Marsh, R. L., Hicks, J. L., Cook, G. I., Hansen, J. S., \& Pallos, A. L. (2003). Interference to ongoing activities covaries with the characteristics of an event-based intention. Journal of Experimental Psychology: Learning, Memory, \& Cognition, 29, 861-870.

McDaniel, M. A., \& Einstein, G. O. (2007). Prospective memory: An overview and synthesis of an emerging field. Thousand Oaks, CA: Sage.
McDaniel, M. A., Guynn, M. J., Einstein, G. O., \& Breneiser, J. (2004). Cue-focused and reflexive-associative processes in prospective memory retrieval. Journal of Experimental Psychology: Learning, Memory, \& Cognition, 30, 605-614.

Pirolli, P., \& CARD, S. (1999). Information foraging. Psychological Review, 106, 643-675.

Schneider, A., Eschman, A., \& Zuccolotto, A. (2002). E-Prime reference guide. Pittsburgh, PA: Psychology Software Tools.

Simon, H. A. (1956). Rational choice and the structure of the environment. Psychological Review, 63, 129-138.

Smith, R. E. (2003). The cost of remembering to remember in eventbased prospective memory: Investigating the capacity demands of delayed intention performance. Journal of Experimental Psychology: Learning, Memory, \& Cognition, 29, 347-361.

Smith, R. E., \& BAYen, U. J. (2004). A multinomial model of eventbased prospective memory. Journal of Experimental Psychology: Learning, Memory, \& Cognition, 30, 756-777.

Smith, R. E., Hunt, R. R., McVay, J. C., \& McConnell, M. D. (2007). The cost of event-based prospective memory: Salient target events. Journal of Experimental Psychology: Learning, Memory, \& Cognition, 33, 734-746.

Tulving, E. (1983). Elements of episodic memory. New York: Oxford University Press.

\section{NOTE}

1. We included a second set of PM cue and PM no-cue conditions in Experiment 1 . The original plan was to examine the influence of cue presentation on task interference using two common PM instruction protocols. In the two PM conditions that we report in Experiment 1, participants were explicitly instructed to make their PM response after their ongoing task response. In the two additional PM conditions that we do not report in Experiment 1, it was not specified to participants whether to make their PM response before or after their ongoing task response. Task interference effects were replicated in these latter conditions. There was significantly less task interference under conditions where expected cues were not presented $(+60 \mathrm{msec})$ than there was where they were presented $(+171 \mathrm{msec})[t(39)=3.29, p<.01]$, and this effect held true for the analysis where only noncue trials presented a minimum of 30 trials away from cue trials were included $[t(39)=2.2, p<.05]$. Further, task interference for the PM no-cue condition $(+60 \mathrm{msec})$ was significantly larger than for the control condition $(-41 \mathrm{msec})[t(38)=$ $4.63, p<.01]$.

(Manuscript received June 14, 2006; revision accepted for publication July 11, 2007.) 